\title{
STABILITY AND BIFURCATION ANALYSIS OF AN OVERHUNG ROTOR WITH ELECTROMAGNETIC ACTUATORS ${ }^{1}$
}

\author{
Piotr M. PrzybyŁowicz, WŁodzimierz Kurnik \\ Warsaw University of Technology, Institute of Machine Design Fundamentals, Warsaw, Poland \\ e-mail: piotr.przybylowicz@pw.edu.pl; wlodzimierz.kurnik@pw.edu.pl
}

\begin{abstract}
A rotating system consisting of a slender massless viscoelastic shaft simply supported in rolling bearings and a rigid massive disc mounted to the overhung end of the shaft is considered to study its stabilization against flutter. Instability and transverse vibration occurs due to the internal friction in the shaft. It is shown in the paper that the disc can be stabilized and its bifurcating self-excited vibration can be effectively reduced and modified by contactless radial magnetic actuators, using two alternative control strategies - semi-active utilizing constant or rotation-dependent actuator voltage or fully active with closed-loop state-dependent feedback. The near-critical transverse disc vibration is analyzed using the theory of Hopf bifurcation. Smooth, soft-type self-excitation is presented after activation of the dynamic vibration control which prevents the system from sudden jumps of vibration amplitude near the critical point.
\end{abstract}

Keywords: overhung shaft, massive disc, flutter, bifurcation, magnetic control

\section{Introduction}

It has been known for almost hundred years that rotors exhibit dynamic instability caused by internal friction in flexible rotating elements or fluid-film dynamic interaction with rigid rotors supported in slide bearings. The mechanism of instability of flutter type is now well recognized and appropriate mathematical tools for analysis adopted and developed (see Osiński et al., 1998, for history and more references). For three decades now attention has been focused on various concepts of stabilization of such systems under increasing rotation speeds and on the near-critical self-excited vibration control first of all to prevent rotors from subcritical unexpected jumps of amplitude. Active stabilization and vibration control of rotors attracts attention of researchers (see Kurnik and Perek, 2015; Przybyłowicz, 2017).

Rotating shafts, even perfectly balanced, may destabilize in form of self-excited vibrations because of the presence of internal friction in the shaft material, structural friction in the supports, joints, etc. Self-excitation occurs when exceeding the critical rotation speed (usually greater than the frequency of the first eigenmode of the shaft treated as a beam) and is characterized by a sudden increase in transverse vibration amplitude. It should be emphasized that this is a completely different phenomenon from whirling of shafts unbalanced by eccentric location of their center of gravity. The deflection line representing the equilibrium position of a horizontally supported shaft lies in a vertical plane while no rotation. A small angular velocity causes the shaft to rotate around this position, gently deflecting the balance plane from the strictly vertical position (in accordance with the direction of rotation). The shaft axis, however, remains stationary. The increasing rotation speed deflects the equilibrium plane more and more from its original position until the internal damping does not allow the shaft material to fully relax the stresses

\footnotetext{
${ }^{1}$ The content of this paper was a part of a presentation at the PCM-CMM-2019 Congress in Cracow.
} 
in one turn, which causes the shaft to perform an additional precession movement with a certain circular frequency (not necessarily equal to the angular velocity) and amplitude. The current equilibrium position changes its form into a qualitatively new oscillating state. This phenomenon is known and referred to as the flutter, i.e. bifurcation of a static equilibrium position into dynamic vibration of periodic nature.

The problem of flutter-type instability of rotating shafts has been a subject of many considerations for a long time. The works by Tondl (1965), Shaw and Shaw (1989) and Muszyńska (2005) deserve special attention in this field. The problem of eliminating this phenomenon, or at least moving it outside the operational range of systems with flexible shafts, has also been addressed by researchers. Structural modifications (changes in stiffness and damping) were available, but those were passive measures incapable of bringing substantial improvement to the system dynamics.

A particularly interesting solution based on the use of active materials exhibiting the shape memory effect was a self-stabilizing shaft made of Nitinol alloy. The flexible shaft when spinning in a deflected equilibrium dissipates energy due to the presence of internal damping. The dissipation of mechanical energy increases the temperature of the alloy the shaft is made of and finally reaches the point of martensitic transformation. This, in turn, leads to a significant change (increase) in Young's modulus and a decrease in the level of internal friction. Both factors have a strong stabilizing effect on the dynamics of the rotating shaft, protecting it from self-excitation. This automatic self-defense mechanism of the system against the dangerous flutter was conceived and presented by Kurnik (1995).

The concept of active stabilization of a rotating shaft discussed in this study is a method based on the use of electromagnetic actuators (Przybyłowicz, 2015, 2017). The actuators are not shaft supports as typical magnetic bearings (explored since the 1950s, see e.g. Schweitzer et al., 1994) but additional elements (Mykhaylyshyn, 2011) contactlessly acting on the disc (or a rimmed fan) mounted rigidly to the elastic shaft by a force of magnetic attraction. The shaft itself is independently supported in mechanical rolling bearings, and a single pair of electromagnetic actuators positioned in opposite is fixed in a close neighbourhood of the disc rim. A similar solution was discussed by Shekhar et al. (2014) who numerically examined the applicability of electromagnetic actuators for active vibration control of long rotors used in power-plant turbines. The authors noticed appreciable vibration reduction with the PD control law related to the difference between nominal and instantaneous air-gap between the rotor surface and actuator poles. Vibration control of a cracked rotor with an electromagnetic actuator was studied by Ebrahimi et al. (2018). They investigated a method of optimal control (with two cost functions) to minimize the vibration and stress at the crack section.

The system presented in this paper is a Jeffcott viscoelastic massless nonlinear shaft with active stabilization, and the source of nonlinearity is the electromagnetic induction. Recently, noteworthy contributions to the nonlinear analysis of Jeffcott rotors have been done by several researchers. Bifurcation analyses of the Jeffcott-rotor system before and after application of positive position feedback control were conducted by Eissa and Saeed (2018). They found an approximate solution to the governing equations by the multiple scales perturbation method and determined optimum working conditions of the control system.

This article focuses on two problems: finding to what extent the applied control based on making use of electromagnetic actuators is efficient in enlarging the safe region of rotating speed, that is increasing the critical threshold (linear analysis) and examining what happens to the system just after the stability is lost and self-excited vibration occurs (nonlinear, bifurcation analysis). 


\section{Analysed system}

Consider an overhung elastic shaft as shown in Fig. 1. Length of the entire shaft is $l$, its overhung part is $a$. Both bearings are perfectly rigid, the rotor terminates with a disc mounted to its right end. In a very close vicinity to the rim of the disc, there is a pair of spatially fixed electromagnets localized in opposite. The electromagnets work independently of each other thus currents in their windings are denoted by $i_{1}$ and $i_{2}$, respectively. The supply voltage yet remains the same and amounts to $U$. Transverse displacement of the shaft at the disc position is $x$ and contributes to the size of the current gap between the rim and the electromagnet core $\Delta_{1}$ and $\Delta_{2}$. The nominal gap (no lateral motion of the shaft) is $\delta$, and its value is assumed to be small enough to consider the magnetic field homogeneous on the one hand, and large enough to prevent the disc from rubbing against the cores while vibration on the other.
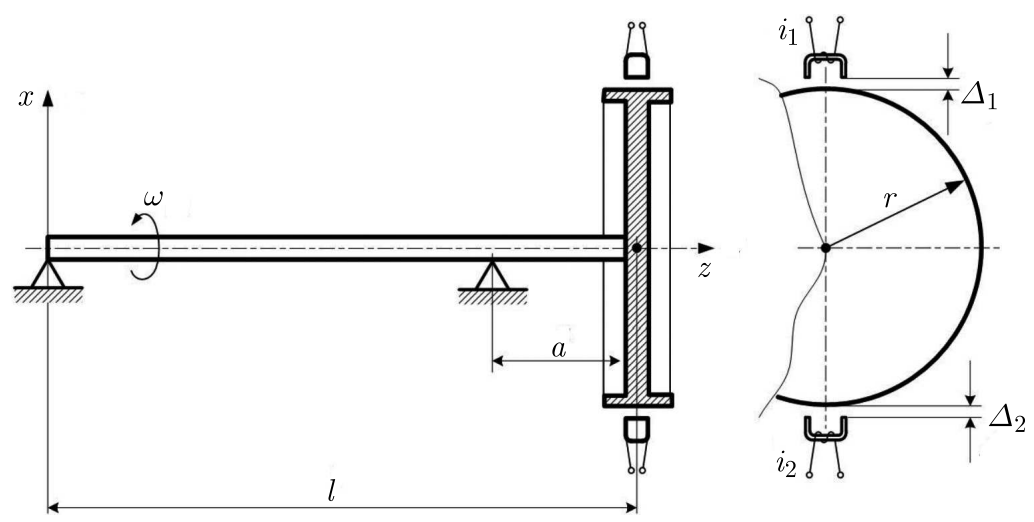

Fig. 1. Model of an overhung shaft with a pair of electromagnets

The shaft rotates with angular velocity $\omega$ and may transversally deform in the perpendicular directions during rotation, see Fig. 2 (displacement in the $O x z$ plane is only disclosed there).

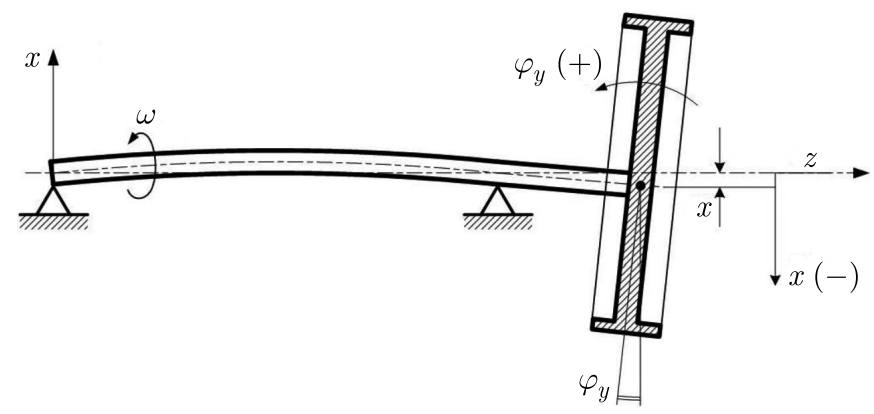

Fig. 2. Shaft undergoing lateral displacement

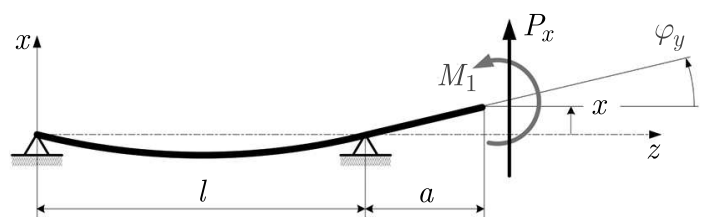

Fig. 3. Transverse force $P_{x}$ and torque $M_{y}$ applied to the disc, resulting in the displacement $x$ and deflection $\varphi_{y}$

The lateral displacement of the center of the disc is denoted by $x$ and $y$, the angles of deflection are $\varphi_{x}$ and $\varphi_{y}$. The disc is perfectly rigid. 
In the following considerations, the shaft is modeled as a discrete massless viscoelastic element to which a disc of mass $m$ and moment of inertia (with respect to diameter) $I_{2}$ is attached at its right overhung end. The system has four degrees of freedom (two transverse displacements and two rotations), the shaft provides stiffness and damping to the disc.

By making use of the method of influence coefficients, the relationships for the lateral displacements and angles of deflection as functions of transverse forces and torques are

$$
\begin{array}{ll}
x=c_{11} P_{x}+c_{12} M_{y} & y=c_{11} P_{y}-c_{12} M_{x} \\
\varphi_{x}=-c_{21} P_{y}+c_{22} M_{y} & \varphi_{y}=c_{21} P_{x}+c_{22} M_{y}
\end{array}
$$

where the influence coefficients are

$$
c_{11}=\frac{a^{3}+a^{2} l}{3 E J} \quad c_{12}=c_{21}=\frac{3 a^{2}+2 a l}{6 E J} \quad c_{22}=\frac{3 a+l}{3 E J}
$$

Consequently, the equations of motion of the disc (transverse motion and rotation) get the following form

$$
\begin{array}{lcc}
m \ddot{x}+h \dot{x}+k_{11} x+k_{12} \varphi_{y}-\beta \omega y=F_{1}-F_{2} & \ddot{y}+h \dot{y}+k_{11} y+k_{21} \varphi_{x}+\beta \omega x=0 \\
I_{2} \ddot{\phi}_{x}+2 I_{2} \omega \dot{\varphi}_{y}-k_{21} y+k_{22} \varphi_{x}=0 & \left.I_{2} \ddot{\varphi}_{y}-2 I_{2} \omega \dot{\varphi}_{x}-k_{12} x+k_{22} \varphi_{y}=0\right)
\end{array}
$$

where the inverse influence coefficients are

$$
k_{11}=\frac{12 E J}{a^{3}} \frac{3 a+l}{3 a+4 l} \quad k_{12}=k_{21}=-\frac{6 E J}{a^{2}} \frac{3 a+2 l}{3 a+4 l} \quad k_{22}=\frac{12 E J}{a} \frac{a+l}{3 a+4 l}
$$

and where $h$ is the coefficient of external damping, and

$$
\beta=\gamma \frac{E J}{\rho A} \frac{\pi^{4}}{l^{4}}
$$

reflects the internal damping resulting from the presence of internal friction in the shaft material. It assumed that the material obeys Kelvin-Voigt rheological law according to which stress $\sigma$ is a function of strain $\varepsilon$ and its rate

$$
\sigma=E\left(1+\gamma \frac{\partial}{\partial t}\right) \varepsilon
$$

where $\gamma$ is the retardation time of the $\mathrm{K}-\mathrm{V}$ model. In equation (2.5) $E$ is Young's modulus of the shaft, $J$-its cross-section moment of inertia, $\rho$ - density, $A$ - cross-section area, $l$ - length. Symbols $F_{1}$ and $F_{2}$ in Eq. (2.3) denote magnetic forces between the electromagnets and the ferromagnetic disc.

\section{Magnetic forces of attraction}

As mentioned before, the magnetic gap between the disc rim and the core of electromagnetic actuators is small enough to justify simplification of the magnetic field to be homogeneous. In such a case, one can make use of well known formula for the magnetic attractive force acting on the electromagnet armature

$$
F=\frac{B^{2} S}{2 \mu_{0}}
$$

where $B$ is magnetic induction, $S$ - cross-section area of the magnetic core, $\mu_{0}$ - magnetic permeability of vacuum. Assuming that the electromagnets operate within a range of currents 
far from magnetic saturation (linearized $B-H$ characteristic), it is possible to derive an explicit expression for the magnetic induction $B_{k}$ in $k$-th electromagnet $(k=1,2)$ versus current $i_{k}$

$$
B_{1}=\frac{N i_{1} \mu_{0}}{2(\Delta-x)} \quad B_{2}=\frac{N i_{2} \mu_{0}}{2(\Delta+x)}
$$

where $N$ is the number of wire turns, $x$ - displacement of the armature (here, the disc rim), see Fig. 4, $\Delta$ is length of the magnetic circuit

$$
\Delta=\delta+\frac{l_{c}}{2 \mu_{c r}}
$$

in which $l_{c}$ is length of the magnetic core, $\mu_{c r}$ - relative magnetic permittivity of the material the core is made of.

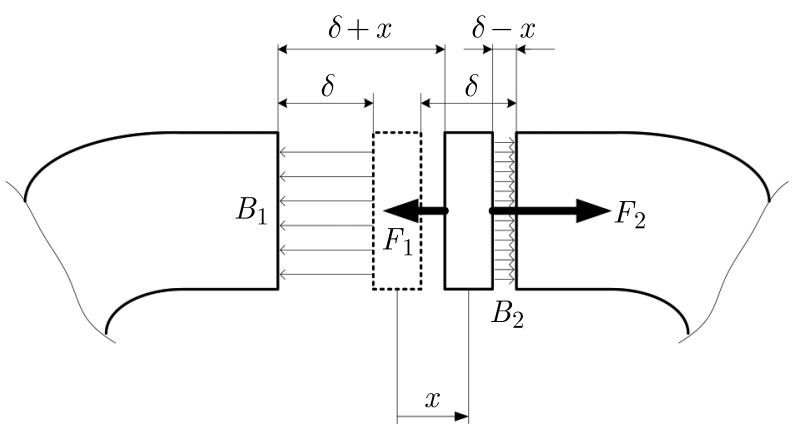

Fig. 4. Nominal gap $\delta$, displacement of the armature (disc rim) $x$ and resulting magnetic forces $F_{1}, F_{2}$

Applying Kirchhoff's second rule to a single electric circuit powering the electromagnet, one writes

$$
U=i_{k} R+N \frac{d \Phi_{k}}{d t}
$$

where $R$ is the electric resistance of the wires, and $\Phi_{k}$ is the magnetic flux in the $k$-th electromagnet $(k=1,2)$. It is described by the equation

$$
\Phi_{k}=\frac{1}{2} N^{2} S \mu_{0} \frac{i_{k}}{\Delta-(-1)^{k} x}
$$

If so, then

$$
U=i_{1} R+\frac{1}{2} N^{2} S \mu_{0} \frac{d}{d t}\left(\frac{i_{1}}{\Delta+x}\right) \quad \text { and } \quad U=i_{2} R+\frac{1}{2} N^{2} S \mu_{0} \frac{d}{d t}\left(\frac{i_{2}}{\Delta-x}\right)
$$

Differentiating Eqs. (3.6), one gets

$$
U=i_{1} R+\frac{N^{2} S \mu_{0}}{2(\Delta+x)^{2}}\left[\frac{d i_{1}}{d t}(\Delta+x)-i_{1} \frac{d x}{d t}\right]
$$

and

$$
U=i_{2} R+\frac{N^{2} S \mu_{0}}{2(\Delta+x)^{2}}\left[\frac{d i_{2}}{d t}(\Delta-x)+i_{2} \frac{d x}{d t}\right]
$$

where the overdots symbolize the first differentiation with respect to time. Finally, the attractive magnetic forces are

$$
F_{1}=\frac{B_{1}^{2} S}{2 \mu_{0}}=\frac{N^{2} S i_{1}^{2} \mu_{0}}{8 \mu_{0}(\Delta-x)} \quad \text { and } \quad F_{2}=\frac{B_{2}^{2} S}{2 \mu_{0}}=\frac{N^{2} S i_{2}^{2} \mu_{0}}{8 \mu_{0}(\Delta+x)}
$$


and their difference (the resultant load exerted upon the disc) is

$$
F_{1}-F_{2}=\frac{N^{2} S \mu_{0}}{8}\left[\frac{i_{1}^{2}}{(\Delta-x)^{2}}-\frac{i_{2}^{2}}{(\Delta+x)^{2}}\right]
$$

From (3.7) and (3.8), one infers that dynamical equations for the currents $i_{k}$ are

$$
\begin{aligned}
& \frac{d i_{1}}{d t}=2 \frac{\left(U-i_{1} R\right)(\Delta-x)}{N^{2} S \mu_{0}}-\frac{i_{1}}{\Delta-x} \frac{x}{d t} \\
& \frac{d i_{2}}{d t}=2 \frac{\left(U-i_{2} R\right)(\Delta+x)}{N^{2} S \mu_{0}}+\frac{i_{2}}{\Delta-x} \frac{x}{d t}
\end{aligned}
$$

As can be seen in the equations describing currents $i_{1}$ and $i_{2}$, there always appears supply voltage $U$ which can be a constant or variable valued quantity. Now it is time to incorporate a strategy to control the performance of the electromagnetic actuators. Basically, one may conceive two approaches to the control - passive (static) and/or active (dynamic) one. Let it be expressed by a simple formula

$$
U=\overbrace{U_{0}+k_{s} \omega}^{\text {static control }} \pm \underbrace{k_{d} \dot{x}}_{\text {dynamic control }}
$$

The static (semi-active) control consists in applying just a constant $U_{0}$ bias voltage to the electromagnetic coils, or it can be extended by adding a proportional term with some gain $k_{s}$. The proportionality may be related to any parameter of the system, but here the natural and obvious choice is the angular velocity $\omega$. On the other hand, the active method should enable the actuators to react to current dynamical changes of the system, i.e. to its state. The state is described by the current position and velocity of the disc. In this work, the control based only on the transverse speed of the disc in the direction $x$ is employed to suppress possible lateral vibration. The differential gain is $k_{d}$.

\section{Stability of the system}

As mentioned in the introduction, fast rotating elastic shafts exhibiting some viscoelastic properties may experience dynamic instability in form of flutter-born whirling due to internal damping. The straight (vertical shafts) or bent due to gravity (horizontal shafts) equilibrium line of the rotor no longer remains at rest and starts to spatially vibrate (rotate) with some whirling velocity $\Omega$. This happens when the rotation speed $\omega$ reaches its critical threshold. The static equilibrium position (straight or bowed) becomes unstable and turns into a new equilibrium state, the oscillating, whirling mode. Such a loss of stability is known as flutter, or Hopf bifurcation.

Find now the critical rotation speed of the considered system by investigating its stability through the eigenproblem of the linearized mathematical model. To this end, introduce new variables $u_{1}, \ldots, u_{10}$ first, to convert four differential equations of motion (2.3) of the second order into eight ones of the first order plus two equations (3.11) for the currents. To enhance the analysis, do it around the equilibrium position for which all new variables are zeros. To achieve this, one should substitute

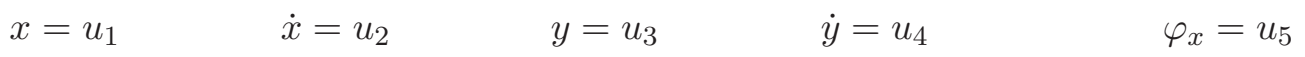

$$
\begin{aligned}
& \dot{\varphi}_{x}=u_{6} \quad \varphi_{y}=u_{7} \quad \dot{\varphi}_{y}=u_{8} \quad i_{1}=u_{9}+\frac{U}{R} \quad i_{2}=u_{10}+\frac{U}{R}
\end{aligned}
$$

In that case, the voltage will be $U=U_{0}+k_{s} \omega \pm k_{d} u_{2}$. 
Rewrite now dynamical equations of motion (2.4) and (3.11) in the new variables

$$
\begin{aligned}
\dot{u}_{1} & =u_{2} \\
\dot{u}_{2} & =-k_{11}^{\prime} u_{1}-h u_{2}+\beta \omega u_{3}-k_{12}^{\prime} u_{7} \\
& +\frac{N^{2} S \mu_{0}}{4 m}\left[\left(\frac{\left.\left.u_{9}+\frac{U_{0}+k_{s} \omega-k_{d} u_{2}}{R}\right)^{2}-\left(\frac{\left.u_{10}+\frac{U_{0}+k_{s} \omega+k_{d} u_{2}}{R}\right)^{2}}{\Delta+u_{1}}\right)^{2}\right]}{\dot{u}_{3}}=u_{4} \quad \dot{u}_{4}=-\beta \omega u_{1}-k_{11}^{\prime} u_{3}-h u_{4}+k_{12}^{\prime} u_{5}\right.\right. \\
\dot{u}_{5} & =u_{6} \quad \dot{u}_{6}=k_{12}^{\prime \prime} u_{3}-k_{22}^{\prime \prime} u_{5}-2 \omega u_{8} \\
\dot{u}_{7} & =u_{8} \quad \dot{u}_{8}=-k_{12}^{\prime \prime} u_{1}+2 \omega u_{6}-k_{22}^{\prime \prime} u_{7} \\
\dot{u}_{9} & =\frac{-2 R r u_{9}}{N^{2} S \mu_{0}}\left(\Delta-u_{1}\right)-u_{2} \frac{u_{9}+\frac{U_{0}+k_{s} \omega-k_{d} u_{2}}{R}}{\Delta-u_{1}} \\
\dot{u}_{10} & =\frac{-2 R r u_{10}}{N^{2} S \mu_{0}}\left(\Delta+u_{1}\right)+u_{2} \frac{u_{10}+\frac{U_{0}+k_{s} \omega+k_{d} u_{2}}{R}}{\Delta+u_{1}}
\end{aligned}
$$

where

$$
k_{i j}^{\prime}=\frac{k_{i j}}{m} \quad k_{i j}^{\prime \prime}=\frac{k_{i j}}{I_{2}} \quad i, j=1,2
$$

see definitions (2.4). Express now the system of ten equations (4.2) in a matrix form by introducing the vector $\mathbf{u}=\left[u_{1}, u_{2}, u_{3}, u_{4}, u_{5}, u_{6}, u_{7}, u_{8}, u_{9}, u_{10}\right]$. A brief form of (4.2) will be then

$$
\dot{\mathbf{u}}=\mathbf{f}(\mathbf{u}, \omega) \quad \text { where } \quad \mathbf{f}(\mathbf{u}, \omega)=\mathbf{A}(\omega) \mathbf{u}+\mathbf{N}(\mathbf{u}, \omega)
$$

in which the right hand side is separated into the linear $\mathbf{A}$ and nonlinear $\mathbf{N}$ part. The presence of $\omega$ in (4.4) emphasizes the role of the angular velocity as the critical bifurcation parameter. The matrix A of the linearized system (round the zero equilibrium position) is as follows

$$
\mathbf{A}(\omega)=\left[\begin{array}{cccccccccc}
0 & 1 & 0 & 0 & 0 & 0 & 0 & 0 & 0 & 0 \\
a_{21} & a_{22} & a_{23} & 0 & 0 & 0 & a_{27} & 0 & a_{29} & a_{210} \\
0 & 0 & 0 & 1 & 0 & 0 & 0 & 0 & 0 & 0 \\
a_{41} & 0 & a_{43} & a_{44} & a_{45} & 0 & 0 & 0 & 0 & 0 \\
0 & 0 & 0 & 0 & 0 & 1 & 0 & 0 & 0 & 0 \\
0 & 0 & a_{63} & 0 & a_{65} & 0 & 0 & a_{68} & 0 & 0 \\
0 & 0 & 0 & 0 & 0 & 0 & 0 & 1 & 0 & 0 \\
a_{81} & 0 & 0 & 0 & 0 & a_{86} & a_{87} & 0 & 0 & 0 \\
0 & a_{92} & 0 & 0 & 0 & 0 & 0 & 0 & a_{99} & 0 \\
0 & a_{102} & 0 & 0 & 0 & 0 & 0 & 0 & 0 & a_{1010}
\end{array}\right]
$$

where the non-zero elements are

$$
\begin{array}{ll}
a_{21}=-\frac{k_{11}}{m}+\frac{N^{2} S \mu_{0}\left(U_{0}+k_{s} \omega\right)^{2}}{m R^{2} \Delta^{3}} & a_{22}=-\frac{h}{2 m}-\frac{N^{2} S \mu_{0} k_{d}\left(U_{0}+k_{s} \omega\right)}{m R^{2} \Delta^{2}} \\
a_{23}=\beta \omega \quad a_{27}=-\frac{k_{12}}{m} & a_{210}=-\frac{N^{2} S \mu_{0}\left(U_{0}+k_{s} \omega\right)}{2 m R \Delta^{2}} \\
a_{29}=\frac{N^{2} S \mu_{0}\left(U_{0}+k_{s} \omega\right)}{2 m R \Delta^{2}} & a_{44}=-\frac{h}{2 m} \quad a_{45}=\frac{k_{12}}{m} \\
a_{41}=\beta \omega \quad a_{43}=-\frac{k_{11}}{m} &
\end{array}
$$




$$
\begin{array}{lcc}
a_{63}=\frac{k_{12}}{I_{2}} & a_{65}=-\frac{k_{12}}{I_{2}} & a_{68}=-2 \omega \\
a_{81}=-\frac{k_{12}}{I_{2}} & a_{86}=2 \omega & a_{87}=-\frac{k_{22}}{I_{2}} \\
a_{92}=-a_{102}=-\frac{U_{0}+k_{s} \omega}{R \Delta} & a_{99}=a_{1010}=-\frac{2 R \Delta}{N^{2} S \mu_{0}}
\end{array}
$$

Having the above matrix defined, one can proceed with the examination of the system stability in terms of the critical threshold (angular speed). To determine its value, resolve the following eigenproblem first

$$
\{\mathbf{A}(\omega)-r \mathbf{I}\} \mathbf{q}=\mathbf{0}
$$

The solution to (4.6) yields five pairs of complex conjugated eigenvalues. The angular speed becomes critical if the real part of one of such pairs exceeds zero and becomes positive (while the rest are negative). In other words, we say $\omega=\omega_{c r}$ when $\max \left\{\operatorname{Re}\left(r_{1}\right), \ldots, \operatorname{Re}\left(r_{10}\right)\right\}=0$. Let the eigenvalue having the maximum real part (at criticality reaching zero) be denoted by $\xi_{\max }$. Analyze now the course of $\xi_{\max }$ in function of quasi-statically increasing angular velocity $\omega$. The moment at which $\xi_{\max }=0$ means the loss of the system stability and the onset of flutter-type self-excited vibration of the disc. This situation is depicted in Fig. 5. Calculations have been carried out for parameters disclosed in Tables 1 and 2.

Table 1. Material and geometrical parameters of the rotating shaft

\begin{tabular}{|l|c|}
\hline Length $l$ & $1.0 \mathrm{~m}$ \\
\hline Diameter $d$ & $0.02 \mathrm{~m}$ \\
\hline Overhung part $a$ & $0.2 \mathrm{~m}$ \\
\hline Density $\rho$ & $7800 \mathrm{~kg} / \mathrm{m}^{3}$ \\
\hline Disc radius $r$ & $0.125 \mathrm{~m}$ \\
\hline Disc thickness $b$ & $0.05 \mathrm{~m}$ \\
\hline Young's modulus $E$ & $2.1 \cdot 10^{11} \mathrm{~N} / \mathrm{m}^{2}$ \\
\hline Retardation of $\mathrm{K}-\mathrm{V}$ model $\gamma$ & $0.0001 \mathrm{~s}$ \\
\hline External damping $h$ & $2.0 \mathrm{~kg} /\left(\mathrm{m}^{2} \mathrm{~s}\right)$ \\
\hline
\end{tabular}

Table 2. More important parameters of the electromagnetic system

\begin{tabular}{|l|c|}
\hline Length of magnetic core $l_{c}$ & $0.2 \mathrm{~m}$ \\
\hline Size of nominal gap $\delta$ & $0.01 \mathrm{~m}$ \\
\hline Number of wire turns & 500 \\
\hline Electric resistance $R$ & $8.0 \Omega$ \\
\hline Relative magnetic permeability of core $\mu$ & 5000 \\
\hline Supply voltage $U_{0}$ & $0-50 \mathrm{~V}$ \\
\hline
\end{tabular}

The course of $\xi_{\max }=\xi_{\max }(\omega)$ shown in Fig. 5 clearly indicates that nearly below $500 \mathrm{rad} / \mathrm{s}$ the system undergoes destabilization $\left(\xi_{\max } \geqslant 0\right)$ and starts to vibrate. The frequency of this vibration is exactly the imaginary part of this eigenvalue. No control has been applied to the system behaving as presented in Fig. 5. This is its own endowed nature determined by the material, environment and the applied assumptions.

Now, look for other (possibly greater) values of the critical rotation speed if some control strategy through electromagnetic forces is turned on in the system. Consider first the simplest approach based only on just activating the electromagnets and supplying them with a constant voltage $U_{0}$. The result is shown in Figs. 6 and 7. 


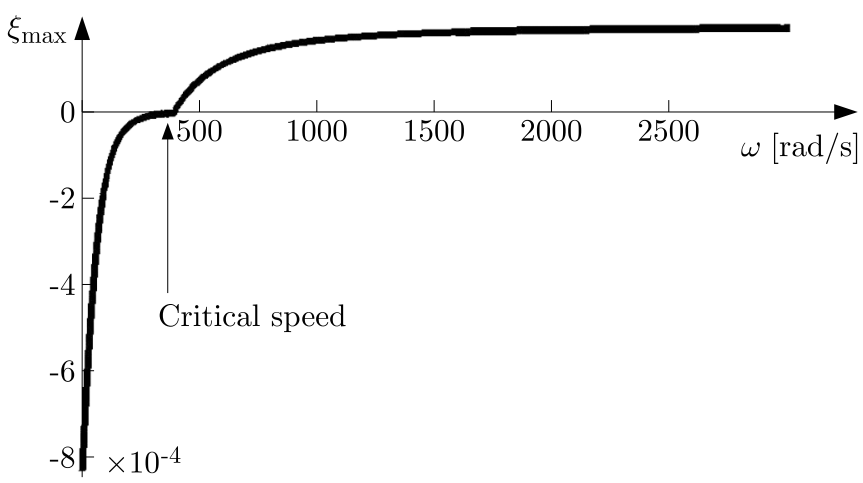

Fig. 5. Evolution of the maximum real part of the eigenvalues (4.6) vs. rotation speed of the shaft
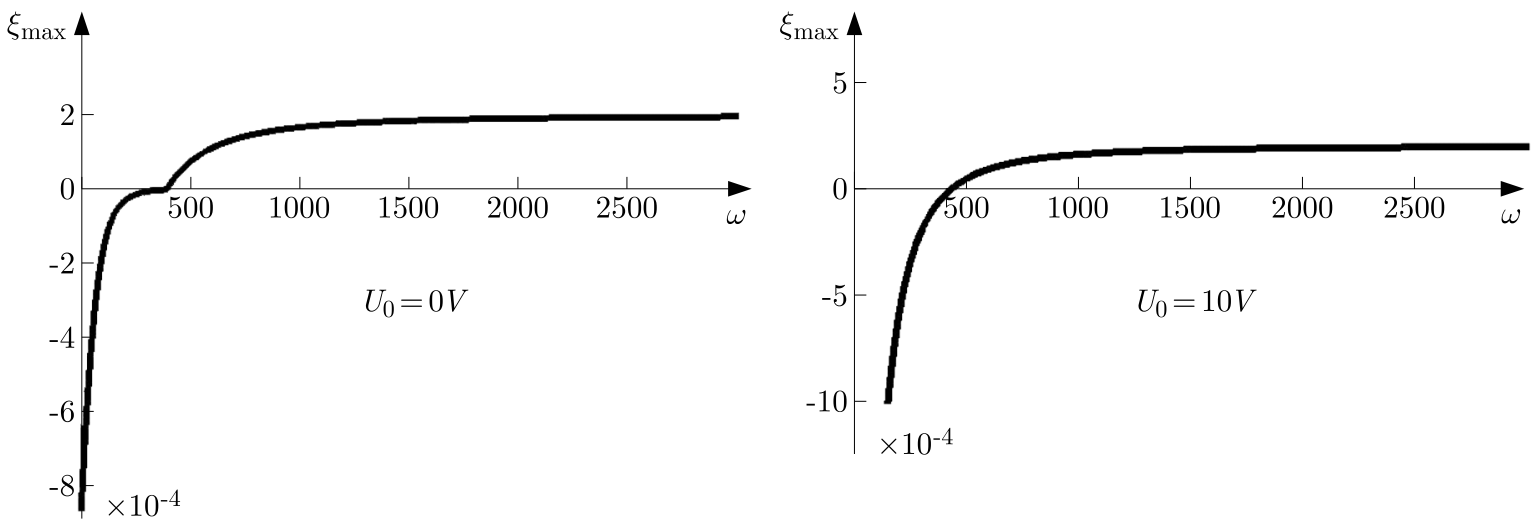

Fig. 6. Critical rotation speed for no control (as in Fig. 5) and static operation of the electromagnets powered by $10 \mathrm{~V}$

(a)

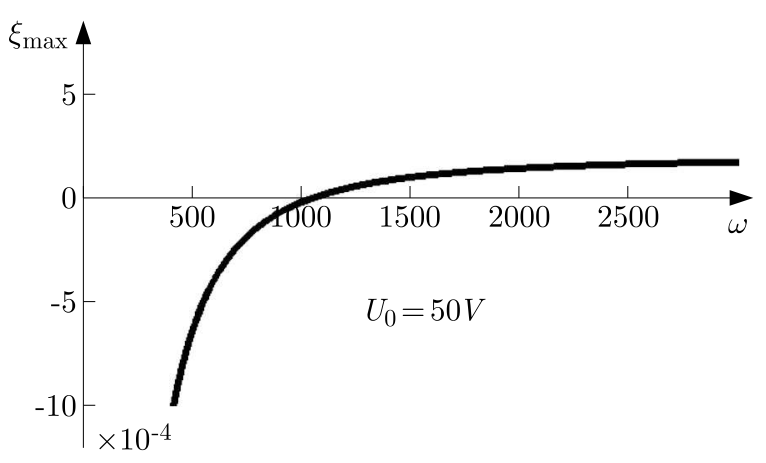

(b) $\xi_{\max }$

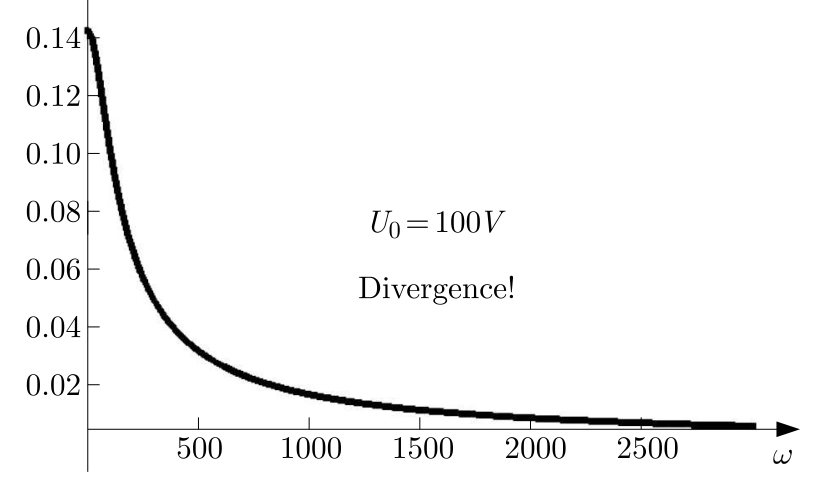

Fig. 7. Critical rotation speed for increased voltage supplying the actuators. The threat of destabilization (in the right picture)

It is observed that the rotating system without any control destabilizes at, roughly, $400 \mathrm{rad} / \mathrm{s}$. Switching on the supply voltage of $10 \mathrm{~V}$ increases the critical rotation speed up to nearly $500 \mathrm{rad} / \mathrm{s}$. Further growth of $U_{0}$ leads to even better results (see left picture in Fig. 7, where $\omega_{c r}$ is about $1000 \mathrm{rad} / \mathrm{s}$ ), but one should bear in mind that such a simple control method has its limitation. The disc entrapped between two electromagnets placed in opposite undergoes greater and greater attracting force with increasing $U_{0}$. The attraction acts in opposite directions thus the central position of the disc is no longer stable. Exceeding a certain value of $U_{0}$ will cause the disc to be attracted to one of the electromagnets and permanent bending of the shaft. Such a situation, which may occur even without rotation, is called divergence and would be a cata- 
strophic loss of stability of the entire system. This, consequently, eliminates this simple method as a safe strategy for stabilizing the shaft.

Much worse results are obtained by another static method of stabilization based on proportional coupling of the supply voltage $U$ with the angular velocity $\omega: U=k_{s} \omega$. Figures 8 and 9 present growth of $\omega_{c r}$ for a few gains $k_{s}$. It is clearly seen the eigenvalue with $\xi_{\max }$ is being chased by another eigenvalue whose real part particularly gains with $\omega$ and finally wins the race becoming a new leader holding the value $\xi_{\text {max }}$. The proportional approach yields poor increments in $\omega_{c r}$ and is very sensitive to divergence at the same time.

(a)

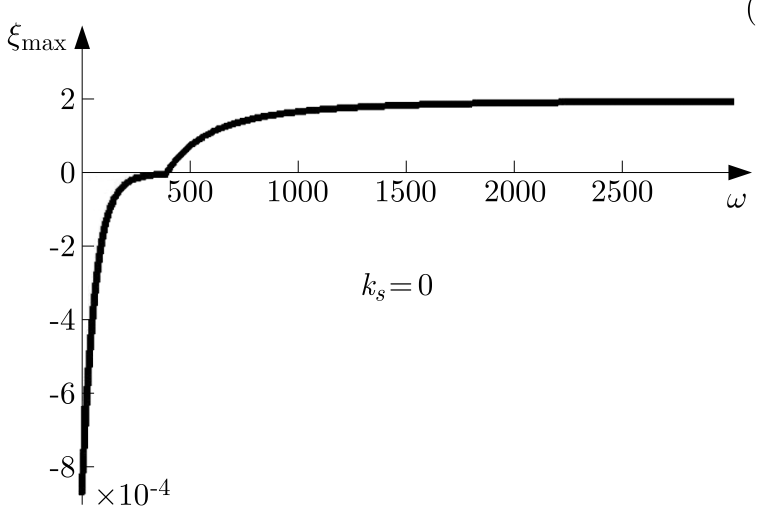

(b)

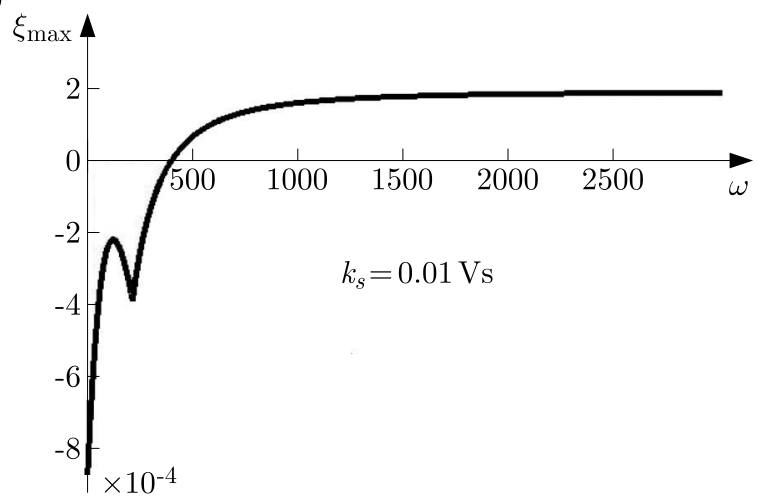

Fig. 8. Proportional feedback. Effect of $k_{s}$ on the course of the real part of the decisive eigenvalue. No visible effect on $\omega_{c r}$

(a)

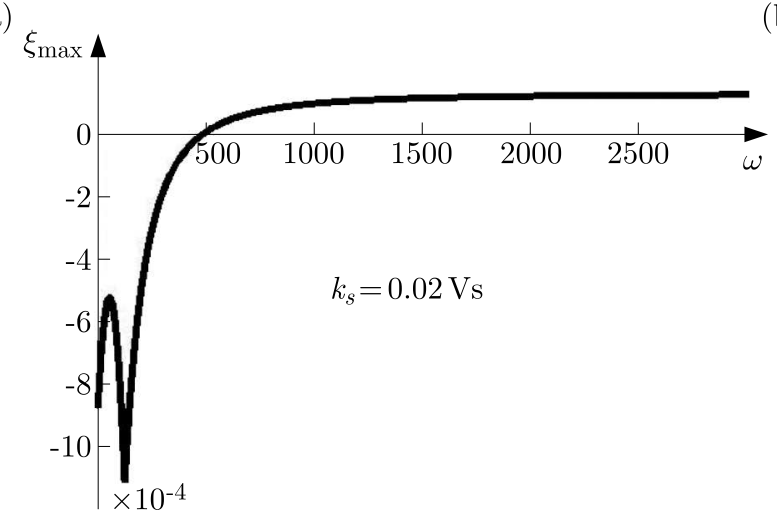

(b)

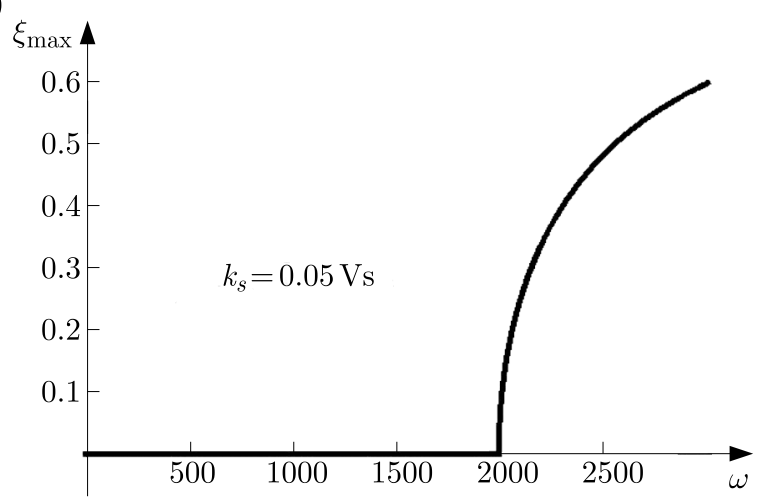

Fig. 9. Proportional feedback leading to a small growth in $\omega_{c r}$ (on the left picture) and inevitable divergence (on the right)

To summarize, among static control strategies, the simple $U_{0}=$ const method offers much better results although it is still burdened with a threat of catastrophic divergence. Expectedly, consider now the dynamic, active approach in which a small and safe $U_{0}$ is supplied to the electromagnets and practically all the voltage comes from electromagnetic induction. By coupling $U$ with the current state of the disc (precisely, its transverse velocity): $U=U_{0} \pm k_{d} \dot{x}$ (the sign \pm depends on whether the disc goes towards the electromagnet or moves away in the opposite direction). The results are disclosed in Figs. 10 and 11.

Two advantageous effects are observed while making use of the active $U=U_{0} \pm k_{d} \dot{x}$ method of stabilization - a significant rise in the critical rotation speed and no danger of disastrous divergence to the system. A summary of the effectiveness of this method is given in Fig. 12 in 
(a)

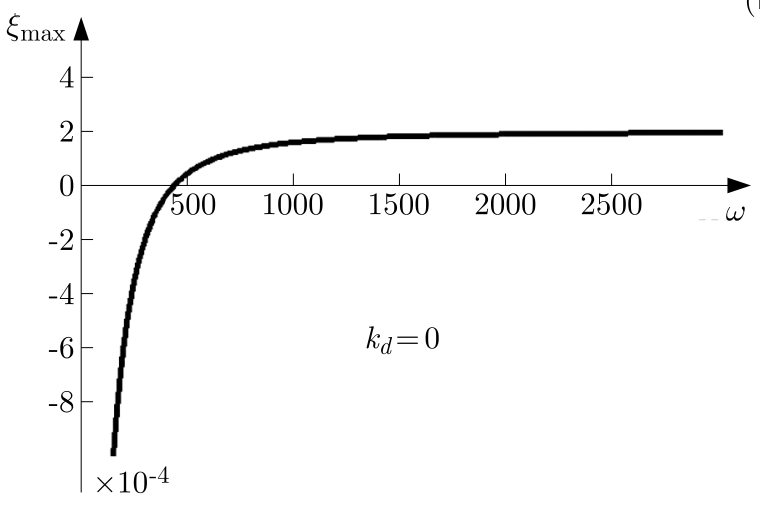

(b)

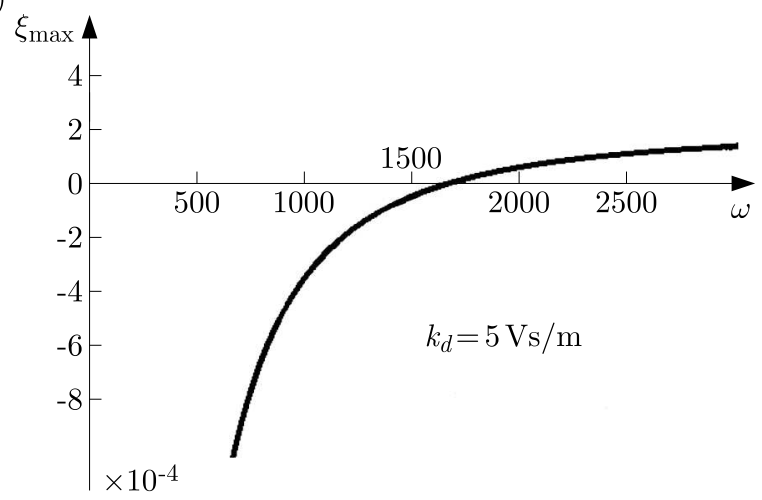

Fig. 10. Velocity-based dynamical control. Large growth of $\omega_{c r}$ for a moderate gain $k_{d}$

(a)

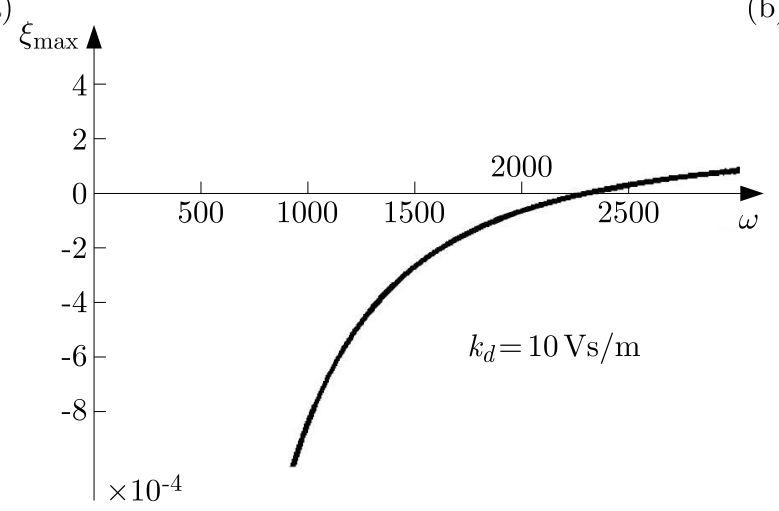

(b)

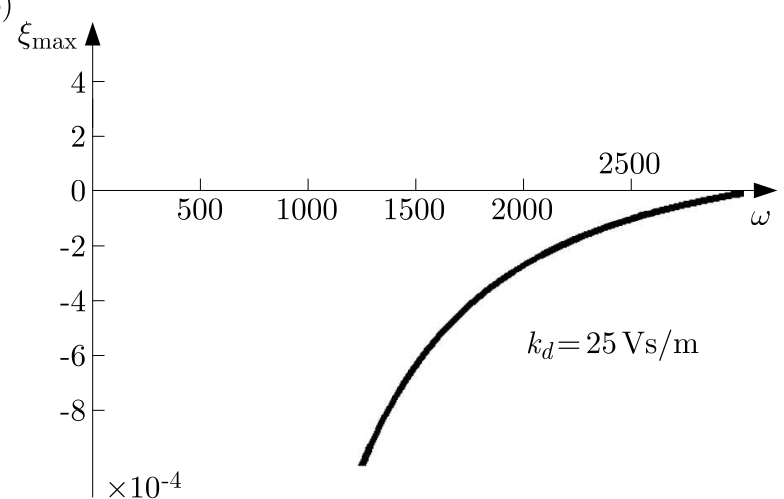

Fig. 11. Further increment in for greater $k_{d}$. No divergence observed

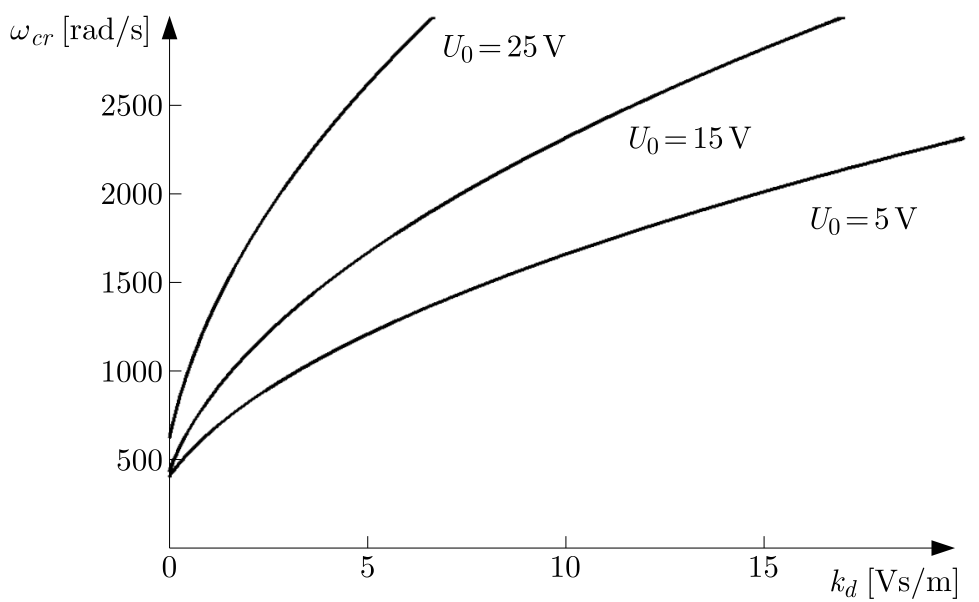

Fig. 12. Critical rotation speed vs. gain in the dynamic control and a few bias voltages

which a rise in $\omega_{c r}$ by several times is clearly noticed. Does it mean that $k_{d}$ can be infinitely increased? Yes, it does. But it would not enlarge the critical speed any more because of magnetic saturation. This is the physical limit of the efficiency of the applied control method incorporating electromagnetic actuators. 


\section{Near-critical behaviour}

After exceeding the critical speed, the system loses its stability. The static equilibrium bifurcates into a new state - flutter-type vibration in form of some limit cycle. Moreover, the cycle itself can be either orbitally stable or not. In the first case, one observes smooth and safe evolution (growth) of the amplitude of self-excited vibration with the increasing rotation speed $\omega$, in the latter there may occur a sudden jump in the vibration amplitude, even below the critical threshold $\omega_{c r}$. That is a much worse and hazardous situation as it may be surprising and threatening to the integrity of the system. To resolve this problem, analyze now the near-critical nonlinear behaviour of the system.

New, oscillating near-critical solution can be found according to the Hopf method. Firstly, conditions satisfying Hopf theorem have to be checked before constructing a bifurcating solution. Return now to the implicit form of equation of motion (4.4) with the right-hand side denoted as $\mathbf{A}(\omega) \mathbf{u}+\mathbf{N}(\omega ; \mathbf{u})=\mathbf{f}(\omega ; \mathbf{u})$. Necessary Hopf conditions require that:

1. The equilibrium position $\mathbf{u}=\mathbf{0}$ is a fixed point of the system of equations, i.e. $\mathbf{f}(\omega ; \mathbf{0})=\mathbf{0}$.

2. Function $\mathbf{f}(\omega ; \mathbf{u})$ is analytical with respect to $\omega$ and $\mathbf{u}$ around $(0 ; \mathbf{0}) \in \Re^{1} \times \Re^{10}$.

3. The matrix $\mathbf{A}$ has a pair of conjugate complex eigenvalues $r(\omega)=\xi_{\max }(\omega)+\mathrm{i} \eta(\omega)$ such that: $\xi_{\max }(0)=0, d \xi_{\max } /\left.d \omega\right|_{\omega=0} \neq 0, \eta(0)=\Omega_{0}>0$.

4. All the rest eigenvalue as of the matrix $\mathbf{A}$ have negative real parts.

5. The nonlinear function fulfills: $\mathbf{N}(0 ; \mathbf{u}) \neq \mathbf{0}$.

Having checked Hopf conditions in the analyzed system, the bifurcating near-critical solution is sought in form of a infinite series in small parameter $\varepsilon$ and containing harmonic functions

$$
\mathbf{u}=\sum_{n=1}^{\infty} \frac{1}{n !} \mathbf{u}^{(n)}(\Omega t) \varepsilon^{n}
$$

where $\mathbf{u}^{(n)}$ are harmonics to be found, and

$$
\Omega=\Omega_{0}+\sum_{n=1}^{\infty} \frac{1}{n !} \Omega_{n} \varepsilon^{n}
$$

is the frequency of the limit cycle also expanded in the power series of $\varepsilon . \Omega_{0}$ is the initial flutter frequency, the terms $\Omega_{n}$ are to be found. To close the solution, one expresses the bifurcation parameter (angular speed here) in form of the analogous series

$$
\omega=\omega_{c r}+\sum_{n=1}^{\infty} \frac{1}{n !} \omega_{n} \varepsilon^{n}
$$

with unknown parameters $\omega_{n}$. Truncating the series $\mathbf{u}$ at the first two terms and applying them to $\mathbf{u}^{(1)}$, one obtains

$$
\omega=\omega_{c r}+\frac{1}{2} \varepsilon^{2} \omega_{2} \Rightarrow \varepsilon=\sqrt{2 \frac{\omega-\omega_{c r}}{\omega_{2}}}
$$

then

$$
\mathbf{u}=\mathbf{u}^{(1)}\left[\left(\Omega_{0}+\Omega_{2} \frac{\omega-\omega_{c r}}{\omega_{2}}\right) t\right] \sqrt{2 \frac{\omega-\omega_{c r}}{\omega_{2}}}
$$

According to the Iooss and Joseph (1980) method, the coefficients $\omega_{2}$ and $\Omega_{2}$ are

$$
\omega_{2}=-\frac{1}{3} \frac{\operatorname{Re}\left\{\Psi_{2}\right\}}{\frac{d \xi_{\max }}{d \omega}} \quad \Omega_{2}=\frac{1}{3} \operatorname{Im}\left\{\Psi_{2}\right\}+\omega_{2} \frac{d \eta}{d \omega}
$$


where the complex number $\Psi_{2}$ is determined from the relation

$$
\Psi_{2}=\frac{3}{2} \sum_{i=1}^{10} \sum_{j=1}^{10} \sum_{k=1}^{10} \frac{\partial^{2} f_{i}\left(\omega_{c r}, 0\right)}{\partial u_{j} \partial u_{k}}\left(q_{j} K_{k}+\bar{q}_{j} L_{k}\right) \bar{q}_{i}^{*}+3 \sum_{i=1}^{10} \sum_{j=1}^{10} \sum_{k=1}^{10} \sum_{l=1}^{10} \frac{\partial^{3} f_{i}\left(\omega_{c r}, 0\right)}{\partial u_{j} \partial u_{k} \partial u_{l}} q_{j} q_{k} \bar{q}_{l} \bar{q}_{i}^{*}
$$

The components of the vectors $\mathbf{K}$ and $\mathbf{L}$ are found from non-singular matrix equations

$$
\begin{aligned}
& \left.\mathbf{K}=-2 \mathbf{A}^{-1}\left(\omega_{c r}\right) \sum_{j=1}^{10} \sum_{k=1}^{10} \frac{\partial^{2} \mathbf{f}\left(\omega_{c r}, \mathbf{0}\right)}{\partial u_{j} \partial u_{k}}\right) q_{j} \bar{q}_{k} \\
& \mathbf{L}=-\left\{\mathbf{A}\left(\omega_{c r}\right)-2 \mathrm{i} \Omega_{0}\right\}^{-1} \sum_{j=1}^{10} \sum_{k=1}^{10} \frac{\partial^{2} \mathbf{f}\left(\omega_{c r}, \mathbf{0}\right)}{\partial u_{j} \partial u_{k}} q_{j} q_{k}
\end{aligned}
$$

where the base vectors $\mathbf{q}$ and $\mathbf{q}^{*}$ come from the eigenproblems

$$
\left\{\mathbf{A}\left(\omega_{c r}\right)-\mathrm{i} \Omega_{0}\right\} \mathbf{q}=\mathbf{0} \quad\left\{\mathbf{A}^{\mathrm{T}}\left(\omega_{c r}\right)+\mathrm{i} \Omega_{0}\right\} \mathbf{q}^{*}=\mathbf{0}
$$

after applying the orthogonality

$$
\sum_{i=1}^{10} q_{i} q_{i}=0
$$

and normalization condition

$$
\sum_{i=1}^{10} q_{i} \bar{q}_{i}=1
$$

The bar over $q_{i}$ denotes complex conjugate. Finally, the first approximation of the bifurcating solution is a harmonic function

$$
\mathbf{u}^{(1)}(\omega ; t)=\operatorname{Re}\left\{\mathbf{q} \exp \left[\mathrm{i}\left(\Omega_{0}+\Omega_{2} \frac{\omega-\omega_{c r}}{\omega_{2}}\right) t\right]\right\}
$$

The most important issue raised at the beginning of this Section is finding out whether the self-excited vibration is orbitally stable or not. Orbital stability means soft, supercritical flutter, relatively safe to the system. Otherwise, the shaft and disc are threatened with subcritical, hard type of self-excitation with jumping vibration amplitude.

The bifurcating near-critical solution is orbitally stable if the Floquet coefficient $\sigma$ remains negative. It can be determined with the accuracy up to reminder of orders higher than four

$$
\sigma(\varepsilon)=\operatorname{Re}\left\{\Psi_{2}\right\} \varepsilon^{2}+O\left(\varepsilon^{4}\right) \quad \text { where } \quad \varepsilon^{2}=2 \frac{\omega-\omega_{c r}}{\omega_{2}}
$$

In Figs. 13 and 14 diagrams of $\sigma$ are shown for all analyzed cases of the applied control, that is both static and dynamic approach. Apparently, and luckily, in each case the Floquet exponents are negative numbers which means that the system is protected from a very dangerous scenario of hard self-excited high-amplitude vibration. The numbers, however, are small. For the static control they converge to zero $\left(U_{0}=\right.$ const $)$, see the left picture in Fig. 13, or may even become positive (the right picture in Fig. 13). Yet before it happens, the system will diverge from the equilibrium (loss of stability in the linear range). Again, much more secure results are given by the dynamic control $\left(U=U_{0}+k_{d} \omega\right)$, see Fig. 14 . 
(a)

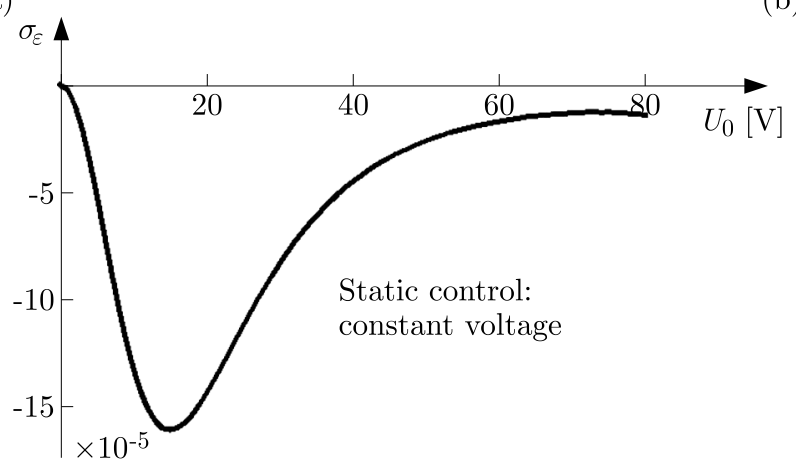

(b)

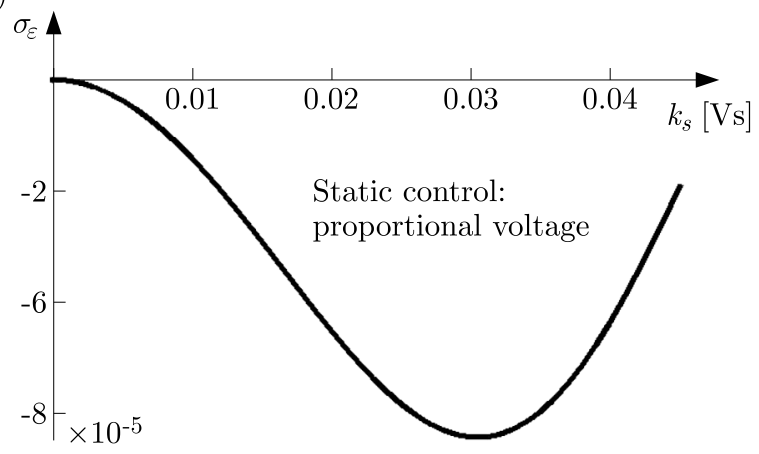

Fig. 13. Floquet exponent of the bifurcating solution in the static control (fixed bias voltage on the left, voltage proportional to rotation speed on the right)

(a)

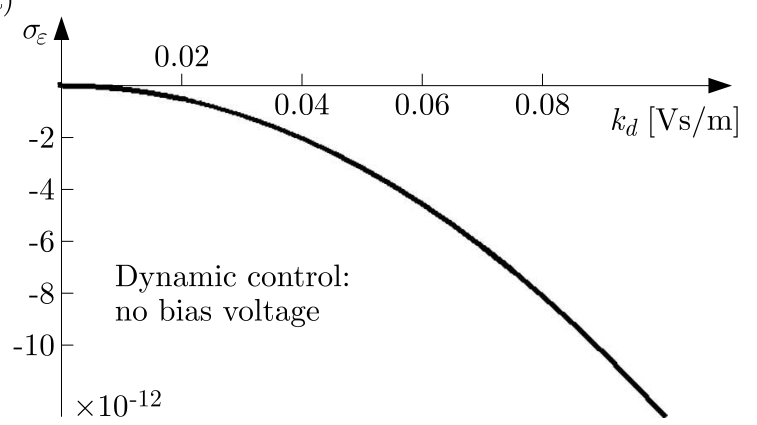

(b)

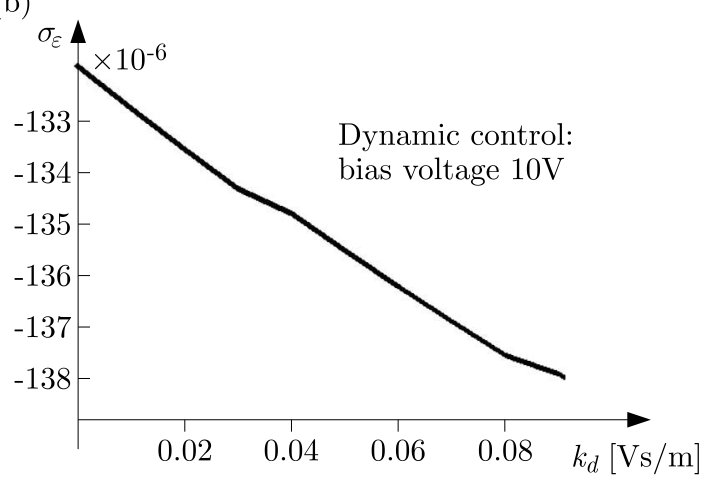

Fig. 14. Floquet exponent in the dynamic control (zero bias voltage on the left, a moderate $U_{0}$ on the right)

\section{Concluding remarks}

The analyzed model is mechanically linear. The source of nonlinearity is electrodynamics only. The examined system is assumed to operate far from the saturation.

The instability is caused by the presence of internal friction in the shaft material. The Kelvin-Voigt rheological model is incorporated to take the internal damping into account. The making use of electromagnetic actuators has proved to be efficient in increasing the critical roation speed. The electromagnets act as external dampers since they dissipate energy in $R L$ circuits. Both control strategies (static and dynamic) are effective, however, the static one may lead to devastating divergence in case of excessive gains. The dynamic approach in which the voltage controlling operation of the electromagnets is a sum of a constant bias voltage and a component proportional to transverse velocity of the laterally vibrating disc has turned out to be particularly efficient as it enables a few fold growth of the critical speed without the danger of devastating divergent loss of stability (rubbing contact of the fast rotating disc with fixed electromagnets).

Both strategies are confirmed to be safe in terms of the nonlinear response as any control method based on operation of electromagnets makes the system supercritical below the saturation threshold (soft and safe type of self-excitation). Nonetheless, this conclusion can only be applied to linear overhung shafts and cannot be extrapolated to rotors exhibiting other nonlinear mechanical effects like von Karman's (progressive) or geometrical (curvature born, degressive) nonlinearity. 


\section{References}

1. Ebrahimi A., Heydari M., Behzad M., 2018, Optimal vibration control of rotors with an open edge crack using an electromagnetic actuator, Journal of Vibration and Control, 24, 1, 37-59, DOI: $10.1177 / 1077546316631880$

2. EIssa M., SAeEd N.A., 2018, Nonlinear vibration control of a horizontally supported Jeffcott-rotor system, Journal of Vibration and Control, 24, 24, 5898-5921, DOI: 10.1177/1077546317693928

3. Iooss G., JosePh D.D., 1980, Elementary Stability and Bifurcation Theory, Springer-Verlag, New York

4. Kurnik W., 1995, Self-stabilization of a composite shaft via thermally adaptive plies, Proceedings of the First International Symposium on Thermal Stresses and Related Topics, Hamamatsu, Japan, 541-544

5. Kurnik W., Perek A., 2015, Kinematically excited vibration of a an asymmetric rotor/bearing system with magnetic lubricant, Machine Dynamics Research, 39, 4, 5-19

6. Muszyńska A., 2005, Rotordynamics, CRC Press, BocaRaton

7. Mykhaylyshyn V., 2011, Application of active magnetic force actuator for control of flexible rotor system vibrations, ETD Archive, Paper 664

8. PrzybyŁowicz P.M., 2015, Stabilization of a rotating shaft by electromagnetic actuators, 86th GAMM Congress, Lecce, Italy, 2015, Book of Abstracts, p. 258

9. PrzybyŁowicz P.M., 2017, Near-critical behavior of an elastic rotating shaft stabilized by electromagnetic actuators, International Journal of Structural Stability and Dynamics, 17, 5, 17400131-15, DOI: 10.1142/S0219455417400132

10. Schweitzer G., Bleuler H., Traxler A., 1994, Active Magnetic Bearings, Zurich, Vdf Hochschulverlag AG.

11. Shaw J., Shaw S.W., 1989, Instabilities and bifurcations in a rotating shaft, Journal of Sound and Vibration, 132, 227-244

12. Shekhar S., Sharma N., Roy H.K., Das A.S., Dutt J.K., 2014, Vibration control of rotor shaft systems using electromagnetic actuator, Mechanisms and Machine Science, 21, DOI: 10.1007/978-3-319-06590-8_116

13. Tondl A., 1965, Some Problems of Rotor Dynamics, Chapman and Hall, London 